\title{
Eficacia de las intervenciones no farmacológicas en la prevención de la depresión posparto: una revisión sistemática de los resultados en gestantes con y sin factores de riesgo
}

\author{
Geraldine Pérez-Miranda* \\ Daniela Ortiz-Pinilla* \\ Daniel Andrés Niño-Mancera* \\ Laura Helena Gómez-Salcedo** \\ Karly Vanessa Bayona-Granados***
}

\begin{abstract}
*Estudiante de Medicina. VII nivel. Universidad Industrial de Santander, Bucaramanga, Colombia **Estudiante de Medicina. X nivel. Universidad Industrial de Santander, Bucaramanga, Colombia

***Médica. Especialista en Psiquiatría. Docente cátedra. Universidad Industrial de Santander, Bucaramanga, Colombia

Correspondencia: Sra. Geraldine Pérez Miranda. Dirección: Cll 30 \# 28-42. Barrio la Aurora, Bucaramanga, Colombia. Correo electrónico: geraldinepmiranda@gmail.com
\end{abstract}

Resumen

Introducción: la depresión posparto es la complicación psiquiátrica más frecuente del periparto, constituye un problema de salud pública debido a consecuencias de corto y largo plazo para madre e hijo. Se considera que el enfoque preventivo tiene gran potencial para reducir su incidencia, así como evaluar la eficacia de las intervenciones según el grupo poblacional al que van dirigidas, contribuye a determinar terapias específicas y disminuir costos en salud. Objetivo: comparar la eficacia de intervenciones no farmacológicas para la prevención de depresión posparto en gestantes con factores de riesgo, respecto a gestantes de la población general. Metodología: se llevó a cabo una búsqueda sistemática en las bases de datos MEDLINE, SCOPUS, CINAHL, EMBASE y LILACS, mediante la cual se identificaron 484 artículos en los idiomas inglés y español, para incluir finalmente, según criterios de elegibilidad y calidad metodológica, un total de 13 ensayos clínicos controlados, que abarcaron una muestra de 5.905 pacientes. Resultados: seis de los siete estudios en gestantes con factores de riesgo impactaron de manera favorable tanto en la disminución de los síntomas como en la disminución del puntaje en las escalas de medición a través del tiempo. En contraste, solo dos de los seis estudios en gestantes de la población general tuvieron impacto significativo. La limitación más importante fue la heterogeneidad de las intervenciones propuestas. Conclusiones: los hallazgos sugieren que las intervenciones preventivas no farmacológicas tienen mayor eficacia cuando son abordadas en gestantes con factores de riesgo y en el periodo posparto. MÉD.UIS.2021;34(1): 73-90.

Palabras clave: Depresión Posparto. Prevención y control. Factores de Riesgo. Psicoterapia. Ejercicio físico. Atención plena. Apoyo social.

\section{Efficacy of non-pharmacological interventions in the prevention of postpartum depression: a systematic review of the results in pregnant women with and without risk factors}

\begin{abstract}
Introduction: postpartum depression is the most frequent peripartum psychiatric complication, it constitutes a public health problem due to short and long-term consequences for mother and child. It is considered that the preventive approach has great potential to
\end{abstract}

Artículo recibido el 20 de noviembre de 2020 y aceptado para publicación el 26 de marzo de 2021. 
reduce its incidence, as well as the evaluation of efficacy in interventions, according to the targeted population group, contributes to determining specific therapies and reducing health costs. Objective: evaluate and compare the efficacy of non-pharmacological interventions for the prevention of postpartum depression in pregnant women with risk factors compared to pregnant women in the general maternal population. Methods: a systematic research was carried out in the MEDLINE, SCOPUS, CINAHL, EMBASE and LILACS databases, through which 484 articles limited to English and Spanish languages were identified, to finally include, according to eligibility criteria and methodological quality, a total of 13 controlled clinical trials, covering a sample of 5,905 patients. Results: six of the seven studies of women with risk factors had a favorable impact on both the decrease in symptoms and the score on the measurement scales over time. In contrast, only two of the six studies in the general population had a significant impact. The most important limitation was the heterogeneity of the proposed interventions. Conclusions: findings suggest that non-pharmacological preventive interventions are more effective in preventing depression when approached in women with risk factors and in the postpartum period. MÉD.UIS.2021;34(1): 73-90.

Keywords: Depression, Postpartum. Prevention and control. Risk Factors. Psychotherapy. Exercise. Mindfulness. Social support.

¿Cómo citar este artículo? Pérez-Miranda G, Ortiz-Pinilla D, Niño-Mancera DA, Gómez-Salcedo LH, Bayona-Granados KV. Eficacia de las intervenciones no farmacológicas en la prevención de la depresión posparto: una revisión sistemática de los resultados en gestantes con y sin factores de riesgo. MÉD.UIS.2021;34(1): 73-90. doi: 10.18273/revmed.v34n1-2021008

\section{Introducción}

El periodo posparto se considera un tiempo de transición desafiante para las madres. Está asociado a cambios psicológicos y fisiológicos que provocan una mayor susceptibilidad al desarrollo de trastornos afectivos, entre los que se encuentra la depresión posparto, complicación psiquiátrica más común de la maternidad ${ }^{1,2}$. Es definida en el Manual Diagnóstico y Estadístico de Trastornos Mentales en su quinta edición (DSM-V) dentro del trastorno depresivo mayor de inicio en el periparto, considerando su desarrollo durante el embarazo o en las primeras cuatro semanas posparto ${ }^{2,3}$. Sin embargo, el período de aparición puede ampliarse de forma variable entre las 4 semanas y los 12 meses después del parto ${ }^{4}$, por lo cual se define alternativamente en la $\mathrm{CIE}-10$ como un trastorno mental y del comportamiento que comienza seis semanas después del parto5. Según la OMS, tiene una prevalencia a nivel mundial de alrededor del $13 \%$ y una incidencia entre el $10 \%$ a $15 \%, 7$. Afecta entre $20 \%$ a $40 \%$ de las mujeres en países de bajos ingresos y al $15 \%$ de mujeres en países de altos ingresos ${ }^{8}$. No existen estudios que describan su prevalencia en Latinoamérica, sin embargo, se han reportado cifras de $22 \%$ en países como Chile y de hasta $32,6 \%$ en México ${ }^{9}$. A nivel nacional, se describe una prevalencia de $12,9 \%{ }^{10}$. Además, se ha asociado a una mayor probabilidad de ideación suicida, siendo el suicidio el responsable del $20 \%$ de las muertes maternas en el posparto"1. Sin embargo, estas estimaciones pueden fluctuar dependiendo de los métodos de diagnóstico utilizados, el tiempo transcurrido desde el nacimiento y el país en el que se realizó el estudio ${ }^{12}$.
Los síntomas más comunes de la depresión posparto son el estado de ánimo triste, dificultad para concentrarse, fatiga, falta de interés por el bebé, sentimiento de ser una mala madre, insomnio, miedo a lastimarse a sí misma o al bebé y pérdida de interés o placer en la vida ${ }^{13}$. Se han descrito también llanto frecuente, falta de apetito, síntomas somáticos múltiples, incapacidad para afrontar situaciones estresantes, baja autoestima e ideación suicida ${ }^{14}$. Así mismo, se ha observado que episodios prolongados producen apego inseguro a sus bebés, madres no emocionalmente disponibles y un mayor reporte de percepciones negativas hacia los mismos ${ }^{15,16}$. Investigaciones observacionales evidencian además que hijos de madres con depresión en comparación con hijos de madres no deprimidas pueden desarrollar un afecto negativo y vínculos menos seguros con sus madres, como también un riesgo mucho mayor de presentar problemas interpersonales, conductuales y $\operatorname{cog}$ itivos ${ }^{17,18}$.

Existen diversos estudios que han identificado el conjunto de factores psiquiátricos, sociales e interpersonales que aumentan el riesgo de las gestantes de desarrollar depresión posparto. Entre los factores psiquiátricos, se encuentran algunos de alto riesgo como los síntomas depresivos prenatales, baja autoestima y ansiedad perinatal, y de riesgo moderado como antecedentes de trastornos depresivos y disforia posparto. Dentro de los factores sociales e interpersonales los eventos de vida estresantes, el bajo nivel de apoyo social y problemas matrimoniales son considerados de riesgo moderado, mientras que el estado civil, bajos ingresos y embarazos no deseados constituyen 
un bajo riesgo ${ }^{19,20}$, al igual que los predisponentes relacionados con la salud materna como el abuso físico, preeclampsia, hiperemesis, complicaciones médicas relacionadas con el parto como cesárea, parto instrumental, parto prematuro y sangrado excesivo durante el parto ${ }^{21}$.

A fin de identificar sistemáticamente a las pacientes con depresión posparto, se utilizan diferentes instrumentos de tamizaje, dentro de estos se encuentra la Escala de Depresión Posparto de Edimburgo (EPDS), una escala de carácter autoinformado y ampliamente validada, diseñada para cuantificar síntomas depresivos cognoscitivos exclusivamente en el posparto, pero actualmente con un uso extendido al periodo prenatal. Consta de 10 ítems cada uno, con puntaje entre 0 a 3, y ha sido traducida a 12 idiomas ${ }^{22,23,24}$. Su sensibilidad varía entre $59-100 \%$ y su especificidad entre $49-100 \% 25$, lo que puede atribuirse a variaciones metodológicas y características poblacionales de los diferentes estudios. El segundo instrumento más usado para este propósito es la Escala de Depresión del Centro de Estudios Epidemiológicos (CESD), diseñada para evaluar sintomatología depresiva en población general pero que ha demostrado incluso identificar más mujeres deprimidas embarazadas o en periodo posparto que la EPDS ${ }^{26,27}$. Su sensibilidad es del $60 \%$ y su especificidad del $92 \% 25$. Otro instrumento utilizado en el mismo marco evaluativo es el Inventario de Depresión de Beck II (BDI-II), que no es normalmente usado para propósitos diagnósticos ni de tamizaje, pero sí es útil para estimar la severidad de los síntomas depresivos, ha demostrado una sensibilidad del $57 \%$ y una especificidad del $97 \%$ para depresión posparto ${ }^{24,25}$. Por otra parte, el Inventario de Predictores de Depresión Posparto Revisado (PDPI-R), usada también dentro de los estudios incluidos en la presente revisión, consiste en 13 predictores y muestra con un puntaje de corte de 10.5 una sensibilidad y una especificidad del $75 \%$ y $54 \%$, respectivamente ${ }^{28}$. Otros instrumentos como la Entrevista Clínica Estructurada para DSM, diseñada para ser administrada por entrevistadores clínicamente capacitados, y el Cuestionario de Riesgo Prenatal (ANRQ), diseñada para evaluar factores estresantes psicosociales tempranos y crónicos, son usadas también en el tamizaje de la depresión posparto $^{29,30}$.

Una forma de reducir la carga de enfermedad que provoca la depresión es disminuir la incidencia de nuevos casos, objetivo que puede lograrse mediante intervenciones preventivas ${ }^{13}$, que implican el tratamiento de un gran número de mujeres de la población general que pueden no estar en riesgo o que involucran a población de alto riesgo ${ }^{31}$. La mayoría de las terapias preventivas disponibles se basan en enfoques no farmacológicos de tipo psicológicos, psicoeducativos, psicosociales y físicos ${ }^{13,32}$. Una de las más estudiadas es la terapia cognitivo conductual, que ha demostrado ser tan eficaz para la depresión posparto como la medicación antidepresiva en los casos leves a moderados y cuando son realizadas desde el periodo antenatal35; tiene como objetivo identificar y cambiar pensamientos distorsionados y enseñar técnicas y habilidades para modificar patrones negativos de comportamiento, mostrando en diferentes estudios su eficacia para reducir síntomas respecto a mujeres no intervenidas ${ }^{15,32-35}$. Así mismo, terapias de psicoeducación también han mostrado reducciones significativas en la incidencia de depresión posparto. Esto mediante clases dirigidas a madres con o sin compañía de sus parejas, que abordan comúnmente temas como la identificación y tratamiento de la depresión, discusiones sobre los desafíos sociales y emocionales del embarazo y educación sobre cuidado personal y habilidades para resolver problemas, además de desmentir creencias poco realistas sobre la maternidad ${ }^{36-38}$. Hace parte también de este enfoque la meditación, atención plena o Mindfulness, adaptada para aumentar la conciencia corporal, la aceptación del cuerpo cambiante y mejorar la conciencia de patrones emocionales y estados mentales propios del embarazo, que en algunos estudios ha demostrado beneficios para la salud materna incluyendo la reducción de síntomas depresivos ${ }^{39-41}$. Por su parte, las terapias psicosociales, que abarcan intervenciones de apoyo social, realizadas mediante estrategias de apoyo mutuo o unidireccional, enfocan sus esfuerzos en mejorar el entorno psicosocial de las madres, modificar determinadas situaciones o el significado que se les da a estas, así como diezmar las reacciones que pueden desembocar los eventos estresantes para la madre ${ }^{42,43}$. También han tenido éxito en la medida en que estructuras sociales más amplias y una mayor disponibilidad percibida de apoyo llevan a valoraciones más benignas de eventos estresantes, evitando una cascada de respuestas emocionales y conductuales negativas ${ }^{42-44}$. De igual manera, las terapias físicas, donde usualmente se incluye la prescripción,, por recomendación del Colegio Estadounidense de Obstetras y Ginecólogos, de ejercicios de leve a moderada intensidad durante al menos 20 a 30 minutos al día mínimo 5 días 
a la semana, contribuyen a un mayor bienestar psicológico materno y menores síntomas depresivos durante el embarazo y después del parto ${ }^{45-47}$.

Mujeres gestantes y en el posparto tienen menos probabilidades de buscar tratamiento para su salud mental en comparación con mujeres no embarazadas, esto es debido a la influencia del estigma que la sociedad le ha asignado históricamente a la salud mental, en añadidura a la generalizada reticencia para la toma de medicamentos psicotrópicos durante la lactancia, a pesar de la evidencia relativa de su seguridad, razón por la cual la depresión es generalmente subtratada ${ }^{48}$. Por consiguiente, y teniendo en cuenta las barreras de atención una vez se constituye la enfermedad y la relevancia de la depresión posparto para la salud pública, así como el alto costo que representa para los sistemas de salud debido a consecuencias a corto y largo plazo, como el desarrollo infantil adverso ${ }^{49}$, se considera que un enfoque de prevención tiene un enorme potencial para reducir la aparición de nuevos casos, sin embargo, los estudios de intervención preventiva son escasos ${ }^{50,51}$. Se sabe poco acerca de las consecuencias económicas y la rentabilidad de las intervenciones destinadas a prevenir o aliviar los síntomas depresivos, pero se han estimado retornos económicos sustanciales para la inversión en la prevención de problemas de salud mental'3,52,53. Actualmente, aunque varias revisiones sistemáticas y metaanálisis han evaluado los efectos de las intervenciones psicológicas en mujeres en riesgo de depresión perinatal, se encuentran pocos estudios que examinen los efectos de intervenciones para la prevención en población general 54 , y menos aún que comparen la eficacia entre estas dos poblaciones. La evidencia disponible demuestra que ensayos que seleccionan participantes según criterios de riesgo tienen más éxito aparente en la prevención de la depresión posparto, así como en la reducción de sintomatología depresiva55. Así mismo, demuestran ser más costo-efectivas que las que incluyen a población general, al comparar su costo en salud por cada evento de depresión que logran evitar ${ }^{56,57}$.

En relación a la definición de eficacia de las intervenciones para prevención de la depresión posparto, se encuentra un patrón en los diversos estudios que la evalúan, en donde, para considerar la intervención como eficaz, se tienen en cuenta tres factores principalmente: cambios en la puntuación de las escalas de tamizaje, la reducción significativa de síntomas depresivos y el periodo en el que estos efectos son logrados ${ }^{56}$. La explicación de los dos primeros reside en que son parámetros de evaluación estándar de la depresión perinatal, el factor tiempo se explica ya que la incidencia de depresión posparto alcanza su pico máximo alrededor del tercer mes, demostrándose que evaluaciones muy prolongadas producen mayores reducciones en el tamaño muestral por pérdidas en el seguimiento de las participantes.

Es así como el objetivo de esta revisión es comparar la eficacia de las intervenciones no farmacológicas para la prevención de depresión posparto y reducción de síntomas depresivos en gestantes con factores de riesgo específicos, respecto a gestantes de la población general.

\section{Materiales y métodos}

Se llevó a cabo una búsqueda sistemática teniendo en cuenta los lineamientos PRISMA, en las bases de datos MEDLINE, SCOPUS, CINAHL, EMBASE y LILACS, bajo diferentes combinaciones de los términos de búsqueda en inglés: "postpartum depression", "perinatal depression", "postnatal depression", "prevention and control", "prevention". Y sus equivalentes en español: "depresión posparto", "depresión post-parto", “depresión postnatal”, "depresión perinatal", "prevención y control", "prevención". La búsqueda se limitó a los idiomas inglés y español. No hubo límite de antigüedad para los estudios y solo se incluyeron ensayos clínicos controlados aleatorizados. (Tabla 1)

Para responder al objetivo de la revisión, se utilizaron criterios de inclusión y exclusión específicos para cada pregunta PICO:

\section{Pregunta PICO \# 1}

En gestantes o en el periodo periparto, de la población general ¿Qué tan eficaces son las intervenciones no farmacológicas para prevenir la aparición de la depresión posparto?
P: gestantes o en el periodo periparto, de la población general
I: intervenciones no farmacológicas
c: ningún tipo de intervención
O: prevenir la aparición de la depresión posparto 


\section{Criterios de inclusión}

- Estudios que evalúen la eficacia de las intervenciones preventivas no farmacológicas para depresión posparto y que evalúen la eficacia de la intervención como resultado principal del estudio.

- Que la población de estudio sean mujeres gestantes o en el periodo periparto.

\section{Criterios de exclusión}

- Estudios que identifiquen los factores de riesgo en la población estudiada.

- Estudios realizados en menores de 18 años.

- Estudios con un periodo de seguimiento menor a dos meses.

- Protocolos de ensayos clínicos.

Tabla 1. Estrategia de búsqueda en bases de datos

*Descriptor MeSH

**Descriptor DeCS

\begin{tabular}{|l|l|l|l|l|l|}
\hline \# & MEDLINE & SCOPUS & CINAHL & EMBASE & LILACS \\
\hline $\mathbf{1}$ & $\begin{array}{l}\text { postpartum } \\
\text { depression }\end{array}$ & $\begin{array}{l}\text { postpartum } \\
\text { depression }\end{array}$ & $\begin{array}{l}\text { postpartum } \\
\text { depression }\end{array}$ & $\begin{array}{l}\text { postpartum } \\
\text { depression }\end{array}$ & $\begin{array}{l}\text { depresión** } \\
\text { posparto }\end{array}$ \\
\hline $\mathbf{2}$ & $\begin{array}{l}\text { perinatal } \\
\text { depression }\end{array}$ & $\begin{array}{l}\text { perinatal } \\
\text { depression }\end{array}$ & $\begin{array}{l}\text { perinatal } \\
\text { depression }\end{array}$ & prevention & $\begin{array}{l}\text { depresión } \\
\text { postparto }\end{array}$ \\
\hline $\mathbf{3}$ & $\begin{array}{l}\text { postnatal } \\
\text { depression }\end{array}$ & $\begin{array}{l}\text { postnatal } \\
\text { depression }\end{array}$ & $\begin{array}{l}\text { postnatal } \\
\text { depression }\end{array}$ & 1 AND 2 & $\begin{array}{l}\text { depresión } \\
\text { postnatal }\end{array}$ \\
\hline $\mathbf{4}$ & $\begin{array}{l}\text { prevention } \\
\text { and } \\
\text { control* }\end{array}$ & $\begin{array}{l}\text { prevention } \\
\text { and } \\
\text { control* }\end{array}$ & prevention & & $\begin{array}{l}\text { depresión } \\
\text { perinatal }\end{array}$ \\
\hline $\mathbf{5}$ & prevention & prevention & $\begin{array}{l}\text { 1 OR 2 } \\
\text { OR 3 }\end{array}$ & & $\begin{array}{l}\text { depresión } \\
\text { post-parto }\end{array}$ \\
\hline $\mathbf{6}$ & $\begin{array}{l}\text { 1 OR 2 } \\
\text { OR 3 }\end{array}$ & $\begin{array}{l}\text { 1 OR 2 } \\
\text { OR 3 }\end{array}$ & 4 AND 5 & & prevención \\
\hline $\mathbf{7}$ & 4 OR 5 & 4 OR 5 & & & $\begin{array}{l}\text { 1 OR 2 OR 3 } \\
\text { OR 4 OR 5 }\end{array}$ \\
\hline $\mathbf{8}$ & 6 AND 7 & 6 AND 7 & & & 6 AND 7 \\
\hline
\end{tabular}

Fuente: elaboración propia de los autores

\section{Pregunta PICO \# 2}

En gestantes o en el periodo periparto, con factores de riesgo para desarrollar depresión posparto ¿Qué tan eficaces son las intervenciones no farmacológicas para prevenir la aparición de la enfermedad?

P: gestantes o en el periodo periparto, con factores de riesgo para desarrollar depresión posparto

I: intervenciones no farmacológicas

C: ningún tipo de intervención

O: prevenir la aparición de la depresión posparto

\section{Criterios de inclusión}

- Estudios que evalúen la eficacia de las intervenciones preventivas no farmacológicas para depresión posparto y que evalúen la eficacia de la intervención como resultado principal del estudio.

- Que la población de estudio sean mujeres gestantes o en el periodo periparto con factores de riesgo para depresión posparto.

\section{Criterios de exclusión}

- Estudios realizados en menores de 18 años.

- Estudios con un periodo de seguimiento menor a dos meses.

- Protocolos de ensayos clínicos.

\section{Resultados}

La estrategia de búsqueda arrojó un total de 484 artículos (MEDLINE = 79), (SCOPUS =169), (CINAHL = 26), (EMBASE $=204)$, (LILACS = 6). Todos los títulos y resúmenes fueron revisados por 2 autores de manera independiente (GP y DP), y se evaluó que respondieran al objetivo de la revisión, seleccionando 68 artículos. Después de eliminar 4 duplicados y con la evaluación de criterios de elegibilidad generales (no responden a ninguna pregunta PICO) mediante la revisión de texto completo, realizada por todos los autores de manera independiente y posteriormente por consenso, se obtuvo un total de 51 artículos para la revisión de las preguntas PICO \#1 y \#2, etapa en la que se clasificaron 11 artículos para la pregunta PICO \#1 y 13 para la pregunta PICO \#2, según criterios de inclusión y exclusión específicos. Finalmente, para evaluar la calidad metodológica y disminuir el riesgo de sesgo de los estudios, se utilizó la lista de chequeo para ensayos clínicos: CONSORT 2010, incluyendo así un total de 13 artículos en la revisión sistemática. (Ver Figura 1)

De los estudios incluidos se extrajeron: datos de publicación (autor (es), año de publicación, país de origen); características de la muestra (tamaño de la muestra, edad, factores de riesgo específicos y otros); características de la intervención (tipo de intervención; proveedor de intervención, modalidad de administración, comienzo, duración y frecuencia) y resultados. Se optó por un abordaje narrativo de los resultados para sintetizar los hallazgos en lugar de un metaanálisis cuantitativo, debido a la homogeneidad no estricta de los estudios en cuanto al enfoque de la intervención, duración del seguimiento y medidas de resultado. (Ver Tabla 2) 


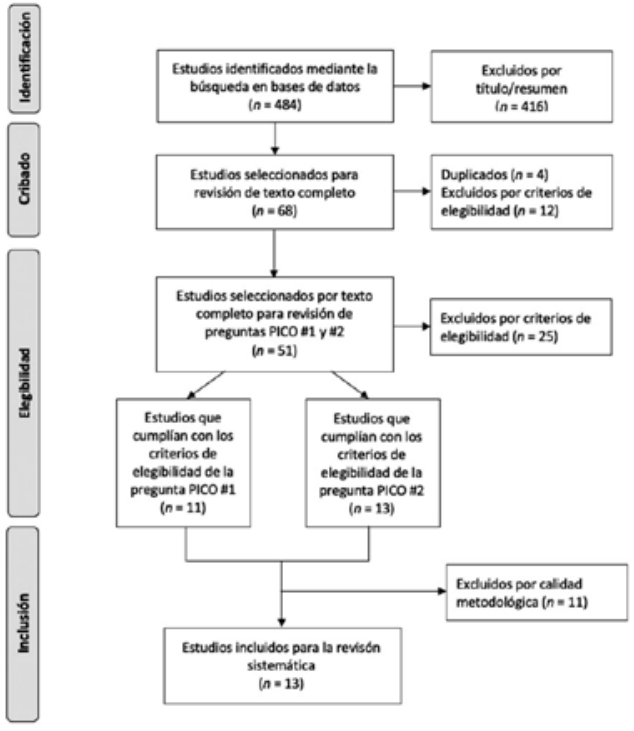

Figura 1. Flujograma de búsqueda

Fuente: elaboración propia de los autores

La tabla 2 muestra las características descriptivas de los estudios. La categoría "control" hace alusión a la terapia ofrecida al grupo control de cada estudio. Se denominó "activo" si fue suministrada dentro del contexto del estudio y era diferente a la del grupo intervención y al tratamiento convencional. "Tratamiento Convencional" hace referencia a que se limitaron solo a dar la atención que generalmente se recibe con respecto al tratamiento y seguimiento médico convencional y "no intervención" como su nombre lo indica, fueron grupos control que no recibieron ningún tipo de atención relacionada. También se tuvo en cuenta el tiempo de intervención, dependiendo si fue en periodo de gestación o posparto. La asignación de riesgo corresponde solo a los estudios en población de riesgo y hace alusión a la escala y el puntaje de corte que las participantes debían obtener para poder ser incluidas. El tiempo de evaluación posparto indica el o los momentos en los que se midieron las variables para la evaluación y seguimiento de la intervención. La administración se refiere a si la terapia requiere que un grupo de madres la practique o si requiere una intervención individual para cada participante.

\section{Características de los participantes}

En general, los estudios se desarrollaron en diferentes países abordando múltiples nacionalidades, por lo tanto, se manejó una amplia variedad de razas y etnias que conllevaron a que, en términos de características sociodemográficas y culturales, no hubiese un patrón constante. La mayoría de los estudios excluyeron pacientes con antecedentes psiquiátricos y/o psicológicos; únicamente Pan et al. ${ }^{58}$, Tandon et al. ${ }^{59}$, Jiang et al. ${ }^{60}$, y Austin et al. ${ }^{61}$ tomaron en cuenta mujeres con dicha condición.

El promedio del tamaño de la muestra en total fue de 454. Solo un estudio, Haga et al..$^{62}$ abordó una población mayor a 1000; cinco: Coll et al. ${ }^{63}$, Songoygard et al. ${ }^{64}$, Dennis et al. ${ }^{65}$, Jiang et al. ${ }^{60}$, Pan et al..$^{58}$, abordaron entre 1000 y 500 pacientes; seis: Fonseca et al. ${ }^{66}$, Austin et al. ${ }^{61}$, Lara et al. ${ }^{67}$, Ngai et al. ${ }^{68}$, Tandon et al. ${ }^{59}$, Pan et al. ${ }^{58}$, entre 500 y 100; y dos: Vismara et al. ${ }^{69}$ y Özkan et al. ${ }^{70}$, menos de 100. Con respecto al antecedente de gestaciones previas, el $85 \%$ de la totalidad de los estudios, tanto en población de riesgo como en general, optó por excluir a las mujeres multigestantes.

\section{Características de las intervenciones}

De las siete intervenciones estudiadas en la población de riesgo, dos corresponden a terapias de psicoeducación: Jiang et al..$^{60}$ y Lara et al. ${ }^{67}$, dos de apoyo social: Vismara et al. ${ }^{69}$ y Dennis et al. ${ }^{65}$, dos a terapia cognitivo conductual: Austin et al. ${ }^{61} \mathrm{y}$ Fonseca et al. ${ }^{66}$, y una estuvo orientada a terapia de ejercicio físico: Özkan et al. ${ }^{70}$ De estas, el $88 \%$ fueron administradas de manera individual y el $71 \%$ se aplicaron en mujeres en periodo posparto. En contraste a dichos resultados, en los estudios de la población sin factores de riesgo no hubo intervenciones de apoyo social, sin embargo, se presentaron las siguientes, una de tipo educacional: Haga et al..$^{62}$, dos terapias de ejercicio físico: Songoygard et al. ${ }^{64}$ y Coll et al. ${ }^{63}$, una de técnicas de meditación: Pan et al..$^{8}$ y dos de terapia cognitivo conductual: Ngai et al. ${ }^{68}$, en donde una de ellas abordó también estrategias de psicoeducación: Tandon et al..$^{59}$ En este grupo el 50\% fueron administrados de forma individual y la otra mitad incluyó sesiones tanto individuales como grupales. Así mismo, el 83\%, que corresponde a la mayoría de las intervenciones, se aplicaron a mujeres en periodo de gestación, a excepción de Haga et al. ${ }^{62}$ quien abordó tanto el periodo preparto como posparto.

\section{Medición de los síntomas}

11 de los 13 estudios incluidos en esta revisión usaron la Edinburgh Postnatal Depression Scale (EPDS) para evaluación y seguimiento en la evolución de los síntomas depresivos. Por el contrario, Lara et al. ${ }^{67}$ y Tandon et al. ${ }^{59}$ emplearon las escalas Beck Depression Inventory II (BDI-II) y la Structured Clinical Interview for the DSM (SCID) con este mismo propósito. 
Eficacia de las intervenciones no farmacológicas en la prevención de la depresión posparto: una revisión sistemática de los resultados en gestantes con y sin factores

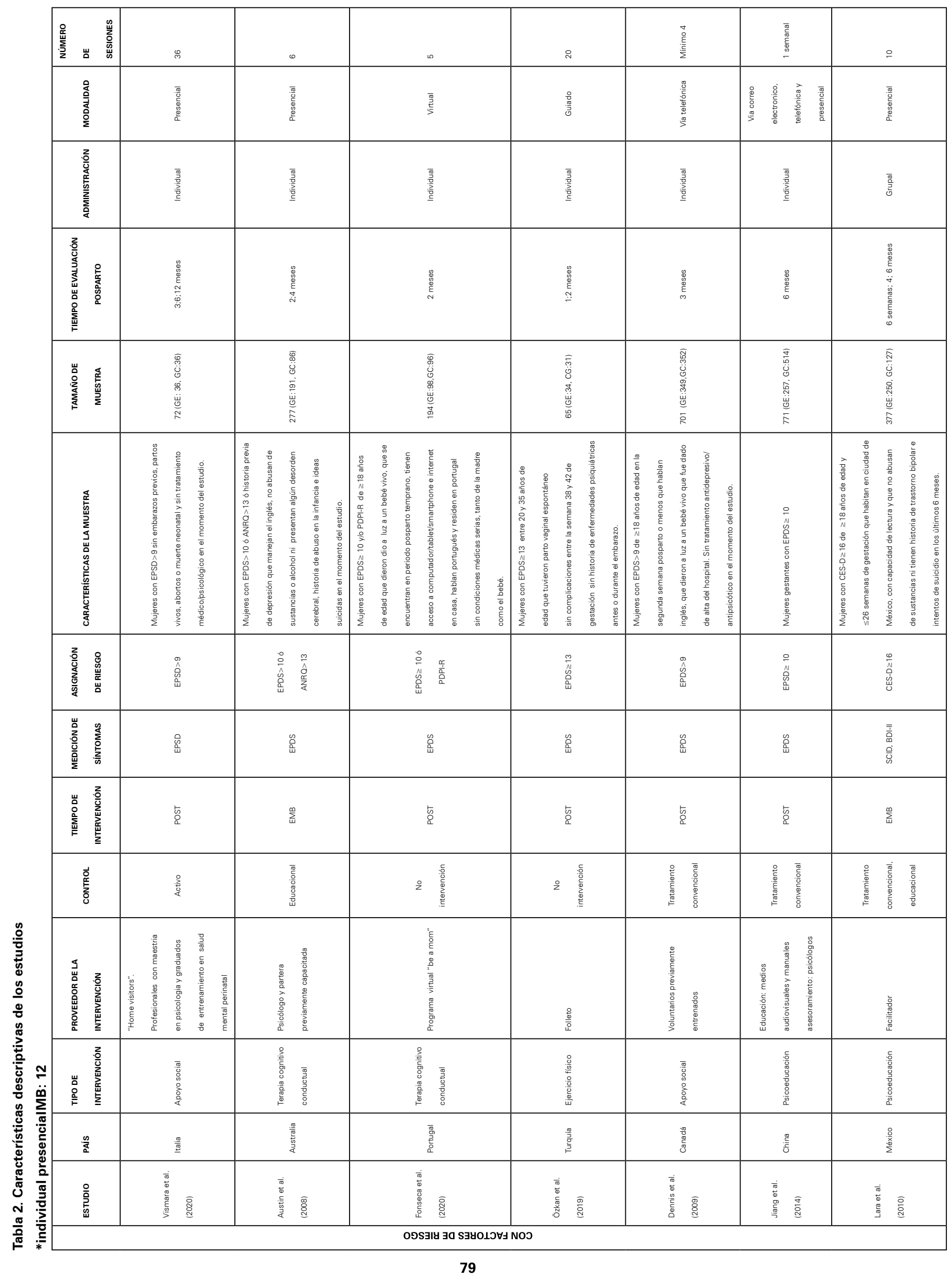


Pérez-Miranda G, Ortiz-Pinilla D, Niño-Mancera DA, Gómez-Salcedo LH,

Bayona-Granados KV

\begin{tabular}{|c|c|c|c|c|c|c|}
\hline 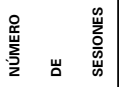 & 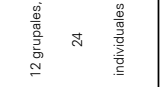 & $\stackrel{g}{q}$ & F & 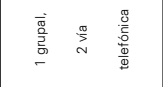 & $\infty$ & $\begin{array}{l}\stackrel{N}{\ddot{\omega}} \\
\stackrel{\dot{\omega}}{\Sigma}\end{array}$ \\
\hline 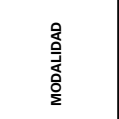 & 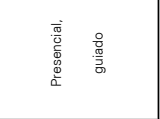 & 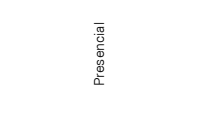 & $\begin{array}{l}\frac{\bar{m}}{\underline{\underline{z}}} \\
\frac{1}{5}\end{array}$ & 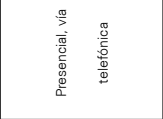 & 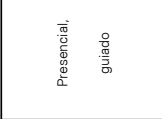 & 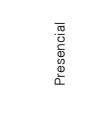 \\
\hline 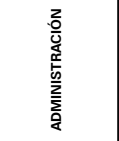 & 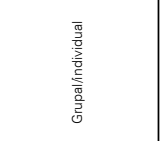 & 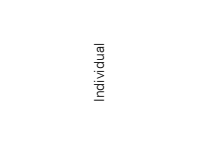 & 縍 & 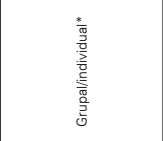 & 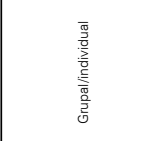 & 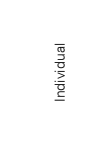 \\
\hline 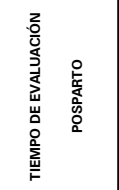 & 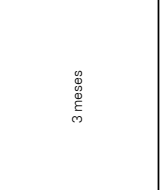 & 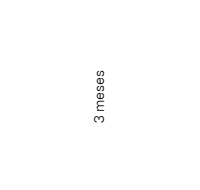 & 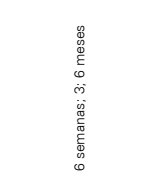 & 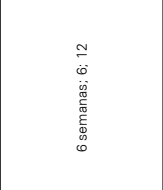 & 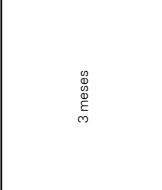 & 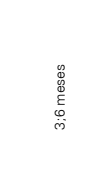 \\
\hline 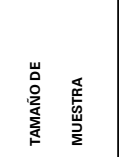 & 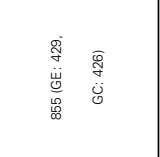 & 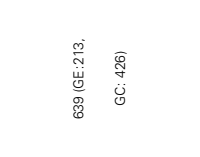 & 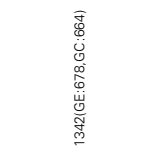 & 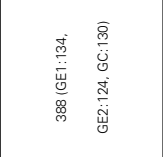 & 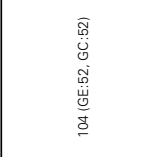 & 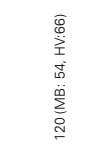 \\
\hline 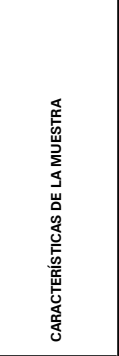 & 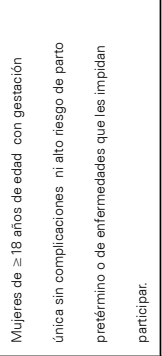 & 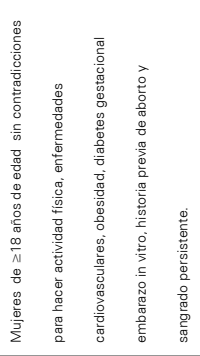 & 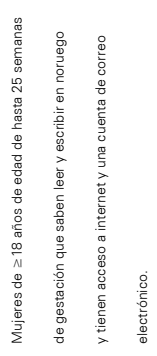 & 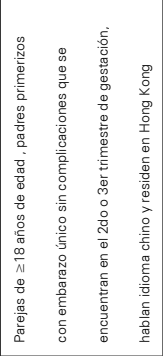 & 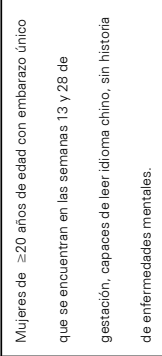 & 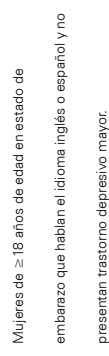 \\
\hline 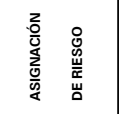 & & & & & , & \\
\hline 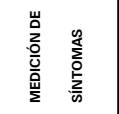 & 㬅 & 总 & 哭 & 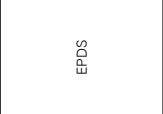 & 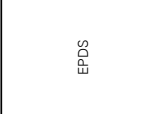 & 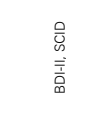 \\
\hline 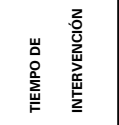 & $\sum_{W}^{\infty}$ & $\sum_{\Psi}^{m}$ & 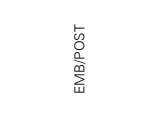 & $\stackrel{\infty}{\stackrel{\infty}{~}}$ & $\stackrel{\infty}{\Psi}$ & $\sum_{=}^{\infty}$ \\
\hline 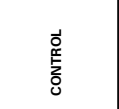 & 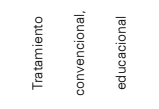 & 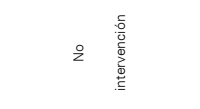 & 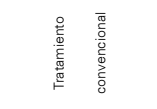 & 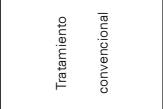 & $\begin{array}{l}\text { 営 } \\
\text { 镸 }\end{array}$ & 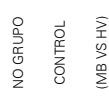 \\
\hline 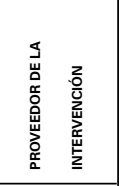 & 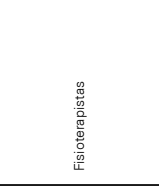 & 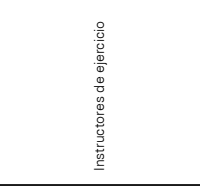 & 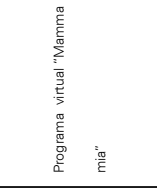 & 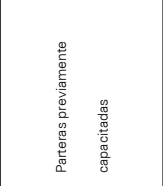 & 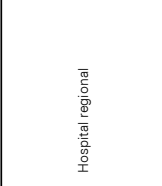 & 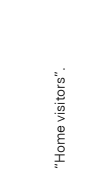 \\
\hline 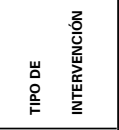 & 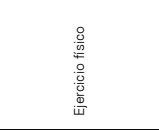 & 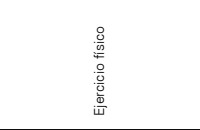 & 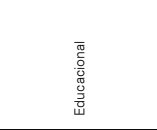 & 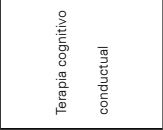 & 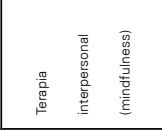 & 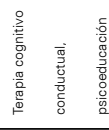 \\
\hline$\frac{0}{\frac{0}{\alpha}}$ & 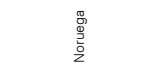 & 离 & 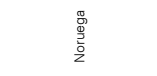 & 骔 & 㞼 & 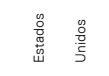 \\
\hline 을 & 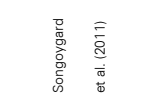 & 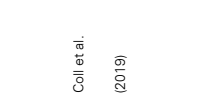 & 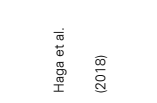 & 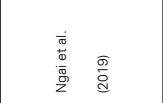 & 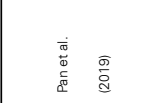 & 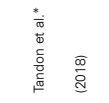 \\
\hline
\end{tabular}




\section{Asignación del riesgo}

Para la asignación del riesgo a la población de estudio se usaron escalas como la EPDS, Antenatal Risk Questionnaire (ANRQ), Postpartum Depression Predictors Inventory-Revised (PDPI-R) y Center for Epidemiologic Studies Depression Scale (CES-D), de las cuales la más usada fue la EPDS, en seis de los siete estudios.

\section{Eficacia de las intervenciones}

En la tabla 3 se resumen los trabajos analizados, separando a la población con factores de riesgo de la población general, detallándose el efecto estadístico y los resultados principales reportados. (Tabla 3 )

\section{Terapia cognitivo conductual}

\section{Población de riesgo}

Para las terapias cognitivo-conductuales (TCC) en población de riesgo, se encontraron dos estudios Austin et al. ${ }^{61}$ y Fonseca et al. ${ }^{66}$ En el estudio de Austin et al. ${ }^{61}$ se evaluó el cambio en los síntomas depresivos, mediante una intervención estructurada de sesiones presenciales de TCC enfocadas en la prevención y el manejo del estrés, la ansiedad y el mal humor en el contexto del embarazo y el cuidado de un nuevo bebé. En comparación al grupo control al que le fue otorgado un folleto educacional. En la última evaluación, al comparar los dos grupos, no se encontraron diferencias significativas en los puntajes de la EPDS. Sin embargo, el análisis de la intervención en el tiempo demostró que los síntomas depresivos se redujeron significativamente desde la pre-intervención hasta la post-intervención y durante el período posnatal $(\mathrm{F}=5.81, p<0.05 ; \mathrm{F}=$ $4.24, p<0.05)$. También se encontró una reducción significativa de los síntomas depresivos en mujeres con puntuaciones más altas (EPDS>12) al inicio de la intervención $(F=115.72, p<0.001 ; F=9.27, p<0.01)$. Ambos resultados fueron independientes del grupo de asignación, por lo que esta intervención no se considera eficaz.

Hallazgos similares fueron reportados en el estudio Fonseca et al. ${ }^{66}$, en donde, por medio de una intervención virtual autoguiada, fundamentada en la combinación de estrategias de la TCC con estrategias que abordaron preocupaciones específicas del período perinatal en comparación a la no intervención, se buscó medir el efecto de la terapia en los síntomas depresivos de la población estudiada. Los resultados no reportaron diferencias significativas entre los grupos al final de las evaluaciones, sin embargo, los cambios entre las puntuaciones de la EPDS de T1 a T2 (periodos de evaluación) fueron significativamente mayores en el grupo experimental $(t=4.95, p<$ $0.001, d=0.623)$, que en el grupo control $(t=3.162$, $p=0.002, d=0.275$ ). De igual manera, un número significativamente mayor de participantes en el grupo experimental mostró una mejoría clínica en el tiempo $\left(X^{2}=10.69, p=0.013\right)$.

\section{Población general}

En relación a las TCC en población general, se incluyeron dos estudios, Ngai et al. ${ }^{68}$ y Tandon et al. ${ }^{59}$ El objetivo de la intervención dirigida por Ngai et al. ${ }^{68}$ fue comparar el efecto de una terapia basada en la pareja, una terapia en mujeres en el periodo periparto y una terapia convencional en el grupo control. La intervención consistió en sesiones grupales durante el periodo antenatal que cubrían una descripción general de los factores estresantes en el período posnatal así como los signos y síntomas de la depresión posparto, técnicas de reestructuración cognitiva, habilidades de resolución de problemas, establecimiento de metas y toma de decisiones y entrenamiento en habilidades de comunicación, reforzadas con sesiones estructuradas vía telefónica en el periodo postnatal. Respecto al riesgo de depresión (EPDS>10), la intervención en parejas demostró una disminución significativa del riesgo, comparado a la intervención en mujeres solamente (MD: $12.495 \%$, IC: 1.1-25.8 $p=0.08$ ), y el grupo de control (MD: 17.8, 95\% IC:3.6-32.0 $p=0.01$ ). Asimismo, el análisis que evaluó el cambio de los síntomas depresivos a través del tiempo reportó una disminución significativa entre la intervención en parejas al compararlo con la intervención en mujeres solamente (MD: $1.46,95 \%$ IC: $0.11-2.81 p=$ $0.03, d=0.26)$ y con el grupo control (MD: $1.71,95 \%$ IC: $0.29-3.13 p=0.01, d=0.3)$. Ambos efectos sólo estuvieron presentes a las 6 semanas posparto y no se mantuvieron a los 6 y 12 meses de la evaluación. Así mismo, estos efectos no se presentaron en el grupo de intervención a mujeres, por lo que no se considera una intervención eficaz por cuestiones de homogeneidad de la revisión. Tampoco hubo una diferencia significativa de las puntuaciones de la EPDS entre los grupos en ningún momento de la evaluación. 
Pérez-Miranda G, Ortiz-Pinilla D, Niño-Mancera DA, Gómez-Salcedo LH,

Bayona-Granados KV

MÉD.UIS. 2021;34(1):73-90

Tabla 3. Resumen de la evidencia

\begin{tabular}{|c|c|c|c|c|}
\hline POBLACIÓN & ESTUDIO & $\begin{array}{c}\text { EFECTO } \\
\text { EVALUADO }\end{array}$ & $\begin{array}{l}\text { EFECTO PRINCIPAL } \\
\text { REPORTADO }\end{array}$ & RESULTADOS PRINCIPALES \\
\hline \multirow{7}{*}{$\begin{array}{l}\text { CON } \\
\text { FACTORES } \\
\text { DE RIESGO }\end{array}$} & $\begin{array}{l}\text { Vismara et al. } \\
\text { (2020) }\end{array}$ & $\begin{array}{l}\text { Síntomas } \\
\text { depresivos }\end{array}$ & $\begin{array}{l}\mathrm{Gl}:[\mathrm{F}(2,70)=40.34, p< \\
0.001 ;(\eta 2 \mathrm{p}=0.07] \\
\mathrm{GC}:[\mathrm{F}(2,70)=7.8, p<0.01 \\
(\eta 2 \mathrm{p}=0.28]\end{array}$ & $\begin{array}{l}\text { Hubo una reducción significativamente mayor a través del tiempo, en los } \\
\text { niveles de depresión, ansiedad y estrés entre las madres pertenecientes al } \\
\text { grupo de intervención, en comparación al grupo control. }\end{array}$ \\
\hline & Austin et al. (2008) & $\begin{array}{l}\text { Síntomas } \\
\text { depresivos }\end{array}$ & $\begin{array}{l}\text { Tendencia lineal: }(\mathrm{F}=5.81 \\
p<0.05) \\
\text { Tendencia cuadrática: }(\mathrm{F}= \\
4.24, p<0.05)\end{array}$ & $\begin{array}{l}\text { Los síntomas depresivos se redujeron significativamente desde la pre } \\
\text { intervención hasta la post intervención y durante el período posnatal, } \\
\text { independiente del grupo de asignación. }\end{array}$ \\
\hline & $\begin{array}{l}\text { Fonseca et al. } \\
\text { (2020) }\end{array}$ & $\begin{array}{l}\text { Síntomas } \\
\text { depresivos }\end{array}$ & $\begin{array}{l}\mathrm{GI}:(\mathrm{t}=4.95, p<0.001, \mathrm{~d} \\
=0.623) \\
\mathrm{GC}:(\mathrm{t}=3.162, p=0.002, \mathrm{~d} \\
=0.275) \\
(\mathrm{X} 2=10.69, p=0.013)\end{array}$ & $\begin{array}{l}\text { No se encontraron diferencias significativas entre los grupos en el último } \\
\text { periodo de evaluación, sin embargo, los cambios entre las puntuaciones de la } \\
\text { EPDS de T1 al T2 fueron significativamente mayores en el GI que en el GC. Un } \\
\text { número significativamente mayor de participantes en el Gl frente al GC mostró } \\
\text { una mejoría clínica en el tiempo (por ejemplo, niveles clínicos de síntomas en } \\
\text { T1 y ausencia de síntomas clínicos en T2). }\end{array}$ \\
\hline & Özkan et al. (2019) & $\begin{array}{l}\text { Síntomas } \\
\text { depresivos }\end{array}$ & $Z=-6.501, p=0.000$ & $\begin{array}{l}\text { Después de } 4 \text { semanas del programa de ejercicio se encontró una mejora } \\
\text { significativa de los síntomas depresivos de GI respecto al GC. }\end{array}$ \\
\hline & Dennis et al. (2009) & Depresión & $\begin{array}{l}\mathrm{T} 1:(\chi 2=12.5, p<0.001 ; \mathrm{RR} \\
=0.46, \mathrm{IC} 0.24-0.62) \\
\mathrm{T} 2:(\chi 2=2.53, \mathrm{p}=0.11)\end{array}$ & $\begin{array}{l}\text { Las mujeres en el GI tuvieron menor probabilidad de presentar depresión } \\
\text { postparto que el GC en la evaluación a las } 12 \text { semanas (T1). No hubo } \\
\text { diferencias significativas a las } 24 \text { semanas (T2). }\end{array}$ \\
\hline & Lara et al. (2010) & $\begin{array}{l}\text { Síntomas } \\
\text { depresivos }\end{array}$ & $\begin{array}{l}{[\chi 2(1)=5.356, p<0.05]} \\
(F=5.11, p=0.02)\end{array}$ & $\begin{array}{l}\text { La incidencia acumulada de depresión en los tres periodos evaluados fue } \\
\text { significativamente menor en el Gl que en el GC. La reducción en los síntomas } \\
\text { depresivos fue significativa independiente del grupo de asignación. }\end{array}$ \\
\hline & Jiang et al. (2014) & $\begin{array}{l}\text { Síntomas } \\
\text { depresivos }\end{array}$ & $(\mathrm{t}=13.059, p<0.001)$ & $\begin{array}{l}\text { Hubo una diferencia significativa en la reducción de síntomas entre los dos } \\
\text { grupos con una mayor reducción en el Gl. }\end{array}$ \\
\hline \multirow{5}{*}{$\begin{array}{l}\text { POBLACIÓN } \\
\text { GENERAL }\end{array}$} & Coll et al. (2019) & $\begin{array}{l}\text { Síntomas } \\
\text { depresivos }\end{array}$ & $\begin{array}{l}\text { (OR: } 0.65 ; 95 \% \mathrm{Cl}: 0.33-1.28) \\
p=0.21 \\
(\mathrm{MD}:-0.6 ; 95 \% \mathrm{Cl}:-1.3-0.1) \\
p=0.1\end{array}$ & $\begin{array}{l}\text { No hubo diferencias significativas en la tasa de depresión postparto, ni en la } \\
\text { reducción de síntomas depresivos entre el Gl y el GC. }\end{array}$ \\
\hline & Haga et al. (2018) & $\begin{array}{l}\text { Síntomas } \\
\text { depresivos }\end{array}$ & $\begin{array}{l}{[F(1)=7.03, p=0.008]} \\
{[F(3)=1.02, p=0.384]} \\
\text { (OR 0.75, 95\% Cl 0.58-0.96, } \\
p=0.024)\end{array}$ & $\begin{array}{l}\text { Se encontró una reducción significativa en la post intervención del GI, } \\
\text { comparado con el GC. No se encontró esta reducción a través del tiempo. La } \\
\text { prevalencia de depresión del Gl fue significativamente menor a la de GC. }\end{array}$ \\
\hline & Ngai et al. (2019) & $\begin{array}{l}\text { Síntomas } \\
\text { depresivos }\end{array}$ & $\begin{array}{l}\text { IP vs M: (MD: } 1.46,95 \% \text { IC: } \\
0.11-2.81 p=0.03, d=0.26) \\
\text { IP vs GC: (MD: } 1.71,95 \% \text { IC: } \\
0.29-3.13 p=0.01, d=0.3) \\
\text { IP vs M:(MD: } 12.495 \%, I C: \\
\text { 1.1-25.8 } p=0.08) \\
\text { IP vs GC: (MD }=17.8,95 \% \\
\text { CI 3.6-32.0, } p=0.01)\end{array}$ & $\begin{array}{l}\text { Los síntomas depresivos se redujeron significativamente en la intervención } \\
\text { de pareja (IP) en comparación a la de mujeres solamente (M) y al GC, en la } \\
\text { semana } 6 \text { postparto. La proporción de mujeres con depresión postparto fue } \\
\text { significativamente menor en el respecto al GC, en la semana } 6 \text {. El efecto de la } \\
\text { intervención no se mantuvo a los } 6 \text { y } 12 \text { meses. }\end{array}$ \\
\hline & Pan et al. (2019) & $\begin{array}{l}\text { Síntomas } \\
\text { depresivos }\end{array}$ & $\begin{array}{l}(F(1,71)=7.36, p=0.008 \\
\eta 2 p=0.09)\end{array}$ & $\begin{array}{l}\text { Se encontraron diferencias significativas en los síntomas depresivos en ambos } \\
\text { grupos a través del tiempo, con una mejoría significativa del Gl comparado con } \\
\text { el GC a los } 3 \text { meses postparto. }\end{array}$ \\
\hline & Tandon et al. (2018) & $\begin{array}{l}\text { Síntomas } \\
\text { depresivos }\end{array}$ & $\begin{array}{l}\mathrm{T} 1:(\beta=-1.56, z=-0.87, p \\
=0.38) \\
\mathrm{T} 2:(\beta=-5.99, z=-2.39, p \\
<0.05)\end{array}$ & $\begin{array}{l}\text { La tasa de cambio en síntomas depresivos y ansiosos entre el Gl y el GC fue } \\
\text { significativo a los } 6 \text { meses postparto, pero no a los } 3 \text { meses. }\end{array}$ \\
\hline
\end{tabular}

Fuente: autores 
En Tandon et al. ${ }^{59}$ se llevó a cabo una TCC que consistió en un programa de visitas domiciliarias estructuradas en un modelo de relaciones positivas entre padres e hijos, apego saludable basado en fortalezas, un enfoque en la familia y la crianza centrada en el desarrollo y el bienestar familiar, con el objetivo de evaluar el efecto de la intervención sobre síntomas depresivos, que en este estudio fueron medidos con la escala BDI-II, encontrándose que la tasa de cambio fue significativa para el grupo experimental respecto al grupo control a los 6 meses posparto $(\beta=-5.99, z$ $=-2.39, p<0.05)$, pero no a los 3 meses $(\beta=-1.56, z=$ $-0.87, p=0.38)$, también se reportó este patrón en los síntomas ansiosos. Otro análisis que midió el efecto del número de sesiones en el desenlace encontró una disminución significativa de los síntomas depresivos en el grupo con mayor número de sesiones [M (SD): $11.0(8.1)]$ vs [M (SD): 6.6 (5.9)].

\section{Terapias psicoeducacionales y educacionales}

\section{Población de riesgo}

Para las terapias psicoeducacionales en población de riesgo se encontraron dos estudios, Jiang et al. ${ }^{60} \mathrm{y}$ Lara et al. ${ }^{67}$ La intervención de Jiang et al. ${ }^{60}$ consistió en un componente educacional comprendido por manuales de información seleccionada sobre depresión posparto, y de dos componentes psicológicos basados en consejería presencial y telefónica especializada acerca de establecimiento de confianza, autoestima y resolución de problemas. Finalmente, se buscó medir el efecto de la intervención en la disminución de los síntomas depresivos, encontrándose una reducción significativa post-intervención $(t=13.059, p<0.001)$.

Asimismo, en Lara et al. ${ }^{67}$ se llevó a cabo una intervención compuesta por sesiones grupales en donde se desarrollaron dos componentes psicológicos dirigidos a aumentar pensamientos positivos, la autoestima y el autocuidado, así como la creación de una atmósfera de confianza y apoyo a través de la dinámica grupal; y de un componente educacional, con secciones de reconocimiento, discusión del período perinatal normal y los factores de riesgo para la depresión posparto. Este estudio encontró que la incidencia acumulada de depresión en los tres periodos evaluados fue significativamente menor en el grupo experimental $\left[\chi^{2}(1)=5.356, p<\right.$ $0.05]$, y que la reducción en los síntomas depresivos fue significativa independiente del grupo de asignación ( $F=5.11, p=0.02)$, sin embargo, no se pudo confirmar un efecto directo de la intervención sobre los resultados obtenidos.

\section{Población general}

Se incluyó Haga et al. ${ }^{62}$, una terapia educacional automatizada de Internet ("Mamma Mia"), con información seleccionada sobre el periodo perinatal, y con el objetivo de evaluar su efectividad sobre los síntomas depresivos perinatales y la incidencia de depresión posparto. En esta intervención no se reportó una reducción significativa de los síntomas depresivos a través del tiempo en el grupo experimental al compararse con el grupo control $[F(3)=1.02, p=0.384]$, sin embargo, sí tuvo un efecto significativo en la disminución del riesgo de depresión posparto (OR 0.75, 95\% Cl 0.58-0.96, $p=$ 0.024), considerándose una intervención eficaz.

\section{Terapia de ejercicio físico}

\section{Población de riesgo}

Respecto a la prevención de la depresión posparto a través de intervenciones que comprendieran ejercicio físico en población con factores de riesgo, se encontró un estudio realizado por Özkan et al. ${ }^{70}$ que tenía como objetivo establecer el efecto de un programa de ejercicio en mujeres con riesgo de depresión posparto. La intervención consistía en un programa de 4 semanas desarrollado a través de investigación en la literatura y recomendaciones de expertos, mientras que el grupo control recibió atención médica estándar postnatal. Los resultados demostraron que la severidad de los síntomas depresivos disminuyó en mayor medida en el grupo experimental comparado con el grupo control a las 4 semanas posterior al inicio de la intervención con una diferencia estadísticamente significativa $(Z=-6.501$, $p=0.001$ ). Comprobando la efectividad del ejercicio durante el periodo posparto, no solo en la reducción de los síntomas depresivos, sino también brindando bienestar psicosocial y reducción en los niveles de ansiedad.

\section{Población general}

Dos estudios que abordaron intervenciones con actividad física se obtuvieron en la búsqueda, el primero fue un estudio llevado a cabo en Noruega por Songøygard et al. ${ }^{64}$ que buscaba demostrar que la ejercitación realizada de forma regular durante el embarazo reducía el riesgo de depresión 
posparto. Las gestantes que formaban parte del grupo experimental recibieron un programa especial balanceado dirigido por fisioterapeutas que comprendía actividad aeróbica con enfoque en la estabilización y ejercitación de la espalda y la pelvis, en tanto que las del grupo control recibieron información prenatal habitual. Los resultados del estudio fueron obtenidos 3 meses después del embarazo y no fueron concluyentes, al no poder demostrar que el ejercicio reducía la severidad de los síntomas depresivos ( $p=0.35$ ) ni la incidencia de mujeres con puntuaciones elevadas en la escala EPDS $(10 \geq O R=0.73$, IC 95\% 0.4-1.5 $\mathrm{p}=0.46 ; 13 \geq \mathrm{OR}=$ 0.44 , IC $95 \% 0.1-1.5 \mathrm{p}=0.25)$. No obstante, aunque la prevalencia de severidad en los síntomas depresivos no difería entre los grupos y no se pudo establecer que la intervención era superior, se encontró un hallazgo conveniente en las mujeres pertenecientes al grupo de intervención que no realizaban ejercicios de forma regular, en quienes se determinó que el riesgo de depresión posparto había disminuido (EPDS score of $\geq 10 ; \mathrm{OR}=0.2$, IC 95\% 0.0-0.9 p = 0.03). Por otra parte, el estudio dirigido por Coll et al. ${ }^{63}$ basado en la actividad física durante el embarazo tenía como propósito evaluar la eficacia de la ejercitación regular antenatal en la prevención de la depresión posparto en un grupo extenso de mujeres gestantes consideradas sanas. Las participantes que recibieron la intervención estuvieron en una sesión de 16 semanas con supervisión, que consistía en actividad aeróbica durante sesiones de 60 minutos a una intensidad de 3 veces por semana. Las participantes del grupo control fueron aconsejadas con mantener las actividades ordinarias de su vida habitual y asistir a los controles de seguimiento médico. Los resultados del estudio evidenciaron que la intervención demostró tener un efecto significativo en la remisión, al disminuir la severidad de los síntomas depresivos prenatales (OR = -0.9, IC 95\%-1.6-0.1, p = 0.02). Aún así, no era efectiva en la reducción de la aparición de depresión posparto 3 meses después del parto (OR $=0.65$, IC 95\% 0.33-1.28, p = 0.21), ni en la reducción de la severidad severidad de los síntomas depresivos (OR=-0.6, IC 95\%-1.3-0.1, p = 0.11).

\section{Apoyo social}

Dentro de la búsqueda general, los programas de apoyo social como instrumento para la prevención de la depresión posparto solo se abordaron en la población de riesgo. Vismara et al. ${ }^{69}$ implementó un programa de visitas domiciliarias orientadas a fomentar una crianza reflexiva, donde se fortalecía la seguridad, apego y sensibilidad materna, además de su capacidad para identificar e interpretar los comportamientos del bebé. Consistió en seis sesiones individuales, impartidas por un profesional, donde se midieron los síntomas antes de la intervención (T1), a los 2 (T2) y a los 4 (T3) meses del periodo posparto. El grupo control recibió atención primaria de rutina, junto con seis llamadas telefónicas, durante los 9 meses de embarazo, por parte de un psicólogo infantil que ofrecía apoyo y asesoría con respecto a dudas sobre la paternidad y comportamientos del niño. Los resultados mostraron que no hubo una diferencia significativa en el puntaje de la EPDS entre el grupo control $(M=8.2)$ y el grupo experimental $(M=7.6)$, sin embargo, sí se observó un efecto a través del tiempo pues tanto el puntaje de la EPDS como los niveles de estrés y de ansiedad mostraron una disminución significativamente mayor entre el grupo control $(G C:[F(2,70)=7.8, p<0.01 ;(\eta 2 p=0.28])$ y el grupo experimental, (GI: $[\mathrm{F}(2,70)=40.34, \mathrm{p}<0.001$; $(\eta 2 p=0.07])$, especialmente en T3. Por otra parte, Dennis et al. ${ }^{65}$ evaluó la efectividad de un programa de apoyo madre-madre vía telefónica que pretendía disminuir el riesgo de depresión a las doce semanas del periodo posparto a partir de la asignación de una madre voluntaria a cada participante. Cada voluntaria fue previamente capacitada con el objetivo de establecer una comunicación fluida con la paciente de manera que represente un apoyo psicológico y emocional. El grupo control tuvo acceso a la atención posparto estándar. El estudio reveló que en la semana doce (T1) de la evaluación, las mujeres en el grupo experimental tenían menos probabilidades de tener depresión posparto que las mujeres en el grupo control, sin embargo, no hubo diferencia significativa en la semana 24 (T2). (T1: $\left[\chi^{2}=12.5, p<0.001 ; R R=\right.$ $0.46, \mathrm{IC} 0.24-0.62])\left(\mathrm{T}_{2}:\left[\chi_{2}=2.53, \mathrm{p}=0.11\right]\right)$.

\section{Técnicas de meditación: Mindfulness}

\section{Población general}

Un programa realizado por Pan et al..$^{8}$ enfocado en salud mental durante el embarazo con una intervención basada en "Mindfulness" que pretendía evaluar el efecto de esta última durante la maternidad temprana fue el estudio que se incluyó para esta terapia. Las intervenciones del grupo experimental que duraron 8 semanas involucraron aprendizaje para manejar los estados emocionales, procesos cognitivos, profundización en el cuidado propio y generación de conciencia de las implicaciones del proceso de parto y la posterior 
crianza. El grupo con el que se comparaba recibió información sobre psicología y fisiología además de prácticas de habilidades durante el embarazo y el periodo posparto. Los resultados fueron obtenidos al tercer mes después del parto y se evidenció una eficacia en el tamaño del efecto a largo plazo para reducir la autopercepción y severidad de los síntomas depresivos $(F=7.36, p=0.008$, partial $\eta 2=0.09)$. Asimismo, se notó simultáneamente una reducción favorable en cuanto a los niveles de estrés, afianzando el beneficio y la aceptabilidad de la intervención.

\section{Discusión}

Esta revisión resume los resultados de 13 ensayos clínicos aleatorizados que fueron llevados a cabo en diez países diferentes involucrando aproximadamente a 5.900 mujeres, bajo diversos contextos y factores sociodemográficos. El proceso de búsqueda resultó en la selección de 13 artículos de los cuales siete se dirigen al abordaje de mujeres con factores de riesgo y seis, a la población general. Se evaluaron diferentes clases de intervenciones no farmacológicas con el fin de determinar la efectividad en la prevención de la depresión posparto de cada una y contrastar el impacto entre poblaciones (población de riesgo y población general).

De los dos estudios que evaluaron los efectos de la TCC en mujeres en riesgo, Austin et al. ${ }^{61} \mathrm{y}$ Fonseca et al., ${ }^{66}$ sólo Austin et al. ${ }^{61}$ reportó un efecto significativamente mayor en la reducción de síntomas depresivos para el grupo experimental; en relación a los estudios que evaluaron la intervención en las mujeres de la población general, Ngai et al. ${ }^{68}$ y Tandon et al.,59 este último también demostrando un efecto protector en el tiempo para el grupo de mujeres intervenidas, mientras que la intervención de Ngai et al. ${ }^{68}$ reportó este efecto en la intervención de parejas, pero no en las mujeres del grupo experimental y solo hasta las 6 semanas posparto.

Estos hallazgos sugieren un efecto similar independientemente de si la intervención se realiza en una población de mujeres con riesgo de depresión posparto o en mujeres de la población general, pero con resultados de mayor magnitud en población con riesgo. Esto corresponde a otras revisiones sistemáticas como las de Werner et al. y Sockol et al., ${ }^{31,71}$ en donde la evidencia sobre la TCC señala una mayor eficacia en la reducción de síntomas depresivos y disminución de incidencia de depresión posparto para las mujeres con riesgo, sugiriendo la importancia de utilizar estos factores predisponentes como criterios de inclusión en intervenciones preventivas.

A pesar de tratarse de terapias en el mismo marco de intervención cognitivo conductual y disminuir la heterogeneidad respecto a características de la población y tiempo de seguimiento, se siguen presentando riesgos de imprecisión, principalmente debido a la variabilidad de las terapias, siendo en Fonseca et al. ${ }^{66}$ una intervención virtual, contrario a Austin et al. ${ }^{61}$, Ngai et al. $^{68}$ y Tandon et al. ${ }^{59}$, en donde la intervención fue presencial, asimismo, la variabilidad en la intervención del grupo control difirió importantemente en Austin et al., ${ }^{61}$ siendo la terapia de control, no convencional, sino de tipo educacional, característica que explicaría el fracaso para demostrar una disminución estadística de los síntomas depresivos al comparar los dos grupos. También se presentaron diferencias importantes por la distribución geográfica de las intervenciones, siendo la población de estudio de Ngai et al. ${ }^{68}$ una población no occidental.

Jiang et al. ${ }^{60} \mathrm{y}$ Lara et al. ${ }^{67}$ fueron los estudios que evaluaron la eficacia de la terapia psicoeducacional y educacional en mujeres con factores de riesgo. Jiang et al. $^{60}$ reportó una respuesta positiva significativa en el grupo de intervención respecto a la reducción de síntomas depresivos, mientras que Lara et al. ${ }^{67}$ reportó una disminución significativa en la incidencia de depresión en el grupo experimental, pero no una reducción de síntomas. A su vez, la terapia educacional en mujeres de la población general, Haga et al. ${ }^{62}$, mostró para el grupo de intervención también una disminución significativa en el riesgo de depresión posparto, pero no una disminución de los síntomas. Esto sugiere un efecto protector importante para la depresión posparto de las terapias psicoeducacionales y educacionales, siendo más evidente en la población de mujeres con factores de riesgo. Esto tiene relación con la eficacia ya comprobada de las terapias psicoeducacionales, como reporta Nagy et al. $^{72}$, en una revisión sistemática sobre depresión en adultos, donde 4 de 17 intervenciones que mostraron reducción significativa en los síntomas depresivos incorporaron estrategias psicoeducacionales.

Dentro de las variaciones más importantes de los estudios, se repite la heterogeneidad respecto a la terapia del grupo de control, siendo en Lara et 
al. ${ }^{67}$ una terapia de tipo educacional, además del tratamiento convencional, que también podría explicar los resultados en la comparación de los grupos. Otra variación importante fue la modalidad de la intervención, virtual en Haga et al. $^{62}$, y una combinación de sesiones presenciales y a distancia en Jiang et al. ${ }^{60}$ y Lara et al. ${ }^{67}$, por último, también puede ser un impedimento al momento de generalización de los resultados, al igual que las TCC, Jiang et al. ${ }^{60}$, un estudio en población no occidental.

De los estudios que evaluaron la eficacia del apoyo social para la prevención de la depresión posparto, Dennis et al. ${ }^{65} \mathrm{y}$ Vismara et al. ${ }^{69}$ no pudieron establecer una relación directa entre el tipo de intervención e incidencia o disminución de los síntomas depresivos por las discrepancias en los aspectos a tratar, mientras Dennis et al. ${ }^{65}$ se enfocó en el apoyo emocional y psicológico de la madre, Vismara et al. ${ }^{69}$ trabajó sobre la relación madre-hijo y su impacto en el desarrollo de la enfermedad. Además, la validez de los resultados de Dennis et. al. ${ }^{65}$ se vio comprometida porque para el diagnóstico clínico de depresión posparto aplicó una entrevista que fue diseñada para ser administrada por un profesional en salud mental cara a cara con el paciente, sin embargo, su aplicación se hizo vía telefónica, fue administrada por un grupo de enfermeras y emplearon una versión resumida del cuestionario, que no ha sido formalmente validada. Adicionalmente, dentro de la discusión, asegura que sus resultados son consistentes con una revisión sistemática que sugiere que para prevención de la depresión posparto es más probable que tenga éxito una intervención administrada individualmente, iniciada en periodo postnatal y dirigida a la población de alto riesgo, sin embargo, el autor de dicha publicación hace parte del grupo de autores del ensayo clínico.

Acerca de Vismara et al. ${ }^{69}$, a pesar de ser el estudio que obtuvo el menor tamaño de muestra entre los 13 ensayos clínicos incluidos en esta revisión, sus resultados concordaron con los de Horowitz et al. ${ }^{73}$, quien, con el doble de la muestra, implementó una intervención bastante similar que consistió en visitas a domicilio a mujeres con factores de riesgo por parte de enfermeras, con el objetivo de mejorar la interacción madre hijo y disminuir la severidad de la depresión posparto. Los hallazgos no reportaron diferencias significativas entre los grupos de tratamiento y de control en cuanto a la eficacia de las relaciones entre la madre y el niño, la capacidad de respuesta al cuidador o la gravedad de los síntomas de depresión posparto, sin embargo, al igual que Vismara et al. ${ }^{69}$ ambos grupos tuvieron una mejora significativa en todas las medidas a lo largo del tiempo.

La búsqueda realizada únicamente arrojó tres ensayos clínicos aleatorizados que evaluaron los resultados a largo plazo de una intervención de ejercicio físico. Los estudios contaban con muestras de pacientes considerablemente grandes, realización de un seguimiento durante un periodo de tiempo adecuado, implementación de una herramienta de tamizaje validada (escala EPDS) y estrategias de cegamiento y aleatorización apropiadas. Las intervenciones basadas en ejercicio físico encontradas en la búsqueda demostraron un efecto con tendencia protectora, pero no significativa para la depresión posparto en población general principalmente. No obstante, se encontró que había un efecto favorable en un subgrupo que no realizaba ejercicio de forma regular en Songøygard et al. ${ }^{64}$, así como también se evidenció un efecto protector asociado a la adherencia al programa durante el periodo prenatal, evaluado por Coll et al. ${ }^{63}$, en contraste a la población con factores de riesgo, donde sí se identificó un efecto beneficioso para las gestantes en el análisis principal, hallazgo reportado en Özkan et al. ${ }^{70}$

Confrontando lo anterior con la literatura actual, estudios como Nakamura et al. ${ }^{74}$ han estipulado que el ejercicio tiene un efecto potencial moderado y conveniente tanto para la prevención, como para el tratamiento de la depresión posparto según Pritchett et al..$^{75}$, aspecto que soporta el resultado de esta revisión discretamente a favor de esta intervención. En cuanto a los síntomas depresivos antenatales y su asociación con la disciplina de actividad física, se ha encontrado y caracterizado el notable efecto en el manejo de la sintomatología antenatal en Daley et al. ${ }^{76}$ Por otra parte, Carter et al. ${ }^{77}$ es un metaanálisis que expone hallazgos que corresponden a los encontrados en esta revisión, en donde se hace evidente el efecto mayor $y$ beneficioso que existe en las mujeres en riesgo de depresión posparto, además de la utilidad de implementar estas intervenciones en gestantes con factores de riesgo sociodemográficos concretamente, según Fontein-Kuipers et al. ${ }^{78}$

En general, la evidencia actual es moderada pero no suficiente para poder establecer con certeza la efectividad de la realización de una rutina de ejercicio para la prevención de la depresión posparto. Aunque 
estudios como Campolong et al. ${ }^{79}$ afirman que existe una favorabilidad y tendencia hacia la instauración de programas y promoción de la actividad física en las gestantes independientemente del trimestre en que se encuentren, se recomienda que la interpretación de estos resultados se realice con cautela, debido a que la evidencia actual respecto a los resultados en la severidad de los síntomas e incidencia de depresión posparto es deficiente por lo que se hace indispensable la intensiva investigación en esta área para poder cubrir las lagunas de conocimiento y así poder determinar un efecto preciso.

Para la sección de intervenciones basadas en "Mindfulness", la búsqueda únicamente obtuvo un estudio en donde se evaluaron gestantes pertenecientes a la población general. Este fue realizado por Pan et al., ${ }^{58}$ y tenía como propósito determinar los efectos de la ejecución de esta intervención durante 8 semanas en este se evidenció la efectividad en la implementación de esta terapia en las gestantes no solo en un efecto moderado de la reducción en el puntaje de la escala utilizada, sino también en la durabilidad del efecto del entrenamiento realizado en la intervención, soportando así la hipótesis planteada por los autores del estudio. Los resultados obtenidos son consistentes con otro metaanálisis que estipula haber encontrado que los programas tenían resultados beneficiosos sobre la psicología materna, Shorey et al. ${ }^{80} \mathrm{Asimismo}$, en esta revisión se resalta la efectividad potencial de la intervención para reducir los niveles de estrés y depresión posparto, así como también fortalecer el vínculo entre la madre y el hijo, similar a Sheffield et al. ${ }^{81}$, Igualmente, se observó que esta intervención, al igual que Taylor et al..$^{82}$ también comprende un beneficio psicológico significativo en la salud mental materna durante el periodo postnatal. La intervención realizada también fue accesible para las gestantes como las instauradas en Smith et al. ${ }^{83}$

Respecto a las limitaciones que pudieron aminorar el efecto de la intervención se debe considerar que en el estudio la tasa de deserción de participantes fue elevada, debido a que se tuvo una pérdida mayor al $20 \%$ de la población inicial, un factor que aumenta el riesgo de sesgo al momento de interpretar los resultados y supone implicaciones sobre los beneficios potenciales de la intervención. Por otra parte, solo un grupo se encontraba cegado de la intervención y los participantes debían realizar actividades en casa las cuales no podían monitorearse con fiabilidad.

La variabilidad en los resultados con respecto al efecto de las terapias sobre la depresión posparto, demostró depender de variables como el tipo de intervención, así como de quien la realice, el tamaño de la muestra y calidad metodológica. Sin embargo, hubo una constante con respecto al tipo de población, de manera que, en las mujeres con factores de riesgo, seis de los siete estudios impactaron de manera favorable, tanto en la disminución de los síntomas en general, como en la disminución del puntaje en las escalas a través del tiempo y la reducción de la incidencia. Por el contrario, para la población general sólo dos de seis estudios impactaron en la disminución del riesgo y severidad de los síntomas. En varios de los estudios incluidos se reporta mayor participación de las mujeres en riesgo, esto se puede explicar por una mayor percepción de la terapia como relevante y útil que se da en estos grupos. ${ }^{61,66}$ También es relevante el diseño de estas intervenciones, ya que la mayoría busca detectar dificultades ya presentes en las gestantes y posteriormente corregirlas, siendo estas dificultades más fácilmente encontradas en las gestantes con perfil de riesgo.

El hecho de que la mayoría de los estudios para la población de riesgo hubiesen intervenido en el periodo posparto y la totalidad de los dirigidos a la población general abordaron a las madres en periodo de gestación, así como el modelo de intervención de predominio individual de las intervenciones en población de riesgo, respecto al grupal o mixto en las de población general, pueden explicar igualmente la relación entre el desenlace y el tipo de población, efectos similares se reportaron en Werner et al. ${ }^{31}$ Para el momento del análisis de los estudios no se encontraron otras revisiones sistemáticas que evaluaran el mismo resultado.

Los resultados de esta revisión son útiles en materia de implementación de intervenciones perinatales preventivas, por parte de instituciones públicas y de los proveedores de atención médica, en cuanto apoyan la importancia de la evaluación del perfil de riesgo y el tamizaje temprano en escenarios como el control prenatal y su posterior derivación, intervención y seguimiento en niveles de mayor complejidad, reduciendo así la incidencia de esta entidad, la carga de la enfermedad y costos para el sistema de salud. 
Respecto a la posibilidad de generalización de los resultados que obtenidos, es pertinente mencionar que no son aplicables en gestantes con riesgos significativos de depresión posparto (ej. Diabetes gestacional) o diagnóstico psiquiátrico establecido (ej. Esquizofrenia), ya que fueron excluidas en la metodología de esta revisión. Por otra parte, los estudios y la búsqueda presentaron numerosas limitaciones, entre ellas se destaca la heterogeneidad en cuanto a las estructuras y direcciones de las intervenciones propuestas, la falta de adherencia al protocolo por parte de las gestantes debido a diversos factores no vinculados y vinculados al embarazo (Gaston et al. ${ }^{84}$ ) junto con la constante pérdida de participantes a lo largo de la duración de los estudios. En cuanto a la búsqueda, se vio limitada por la reducida cantidad de estudios pertinentes realizados en este ámbito y presumiblemente a la restricción del lenguaje (inglés-español).

\section{Conclusiones}

La evidencia de esta revisión sistemática sugiere que las intervenciones preventivas no farmacológicas demuestran una mayor eficacia en la reducción de la incidencia y sintomatología de depresión posparto cuando son abordadas en la población con factores de riesgo, comparado a cuando se aplican en la población en general. Intervenir en el periodo posparto mostró ser más efectivo para la reducción del riesgo y de la severidad de los síntomas en comparación al periodo de gestación. Se recomienda ampliar la investigación sobre la eficacia de las intervenciones preventivas no farmacológicas en poblaciones con características y factores de riesgo diferentes, haciendo énfasis en la estandarización y categorización del tipo de intervención implementada, duración del seguimiento de los participantes, estrategia y métodos de evaluación, para así poder establecer recomendaciones generalizadas acerca del uso de estas intervenciones para la prevención de la depresión posparto.

\section{Referencias bibliográficas}

1. Afshari, P, Tadayon, M, Abedi, P, Yazdizadeh, S. Prevalence and related factors of postpartum depression among reproductive aged women in Ahvaz, Iran. Health Care Women Int. 2019;41(3):1-11.

2. Mendoza C, Saldivia S. Actualización en depresión postparto: el desafío permanente de optimizar su detección y abordaje. Rev. méd. Chile. 2015;143(7):887-894.

3. Asociación Americana de Psiquiatría. Manual Diagnóstico y Estadístico de los Trastornos Mentales, quinta edición (DSM-V). Arlington (VA), EE.UU: American Psychiatric Association Publishing; 2013.
4. Stewart DE, Vigod SN. Postpartum Depression: Pathophysiology, Treatment, and Emerging Therapeutics. Annu. Rev. Med. 2019;70(1):183-196.

5. Norhayati MN, Nik Hazlina NH, Asrenee AR, Wan Emilin WMA. Magnitude and risk factors for postpartum symptoms: A literature review. J Affect Disord. 2015;175(1):34-52.

6. World Health Organization. Maternal mental health. [online] Disponible en: http://www.who.int/mental_health/maternalchild/maternal_mental_health/en/.

7. Barrera AZ, Nichols AD. Depression help-seeking attitudes and behaviors among an Internet based sample of Spanishspeaking perinatal women. Rev Panam Salud Publica. 2015;37(3):148-53.

8. Sefogah PE, Samba A, Mumuni K, Kudzi W. Prevalence and key predictors of perinatal depression among postpartum women in Ghana. Int. J. Gynecol. Obstet. 2020;149:203-210.

9. Romero D, Orozco LA, Ybarra JL, Gracia BI. Sintomatología depresiva en el post parto y factores psicosociales asociados. Rev. chil. obstet. ginecol. 2017;82(2):152-162.

10. Rincón-Pabón D, Ramírez-Vélez R. Postnatal depression in Colombian women: secondary analysis of the 2010 Colombian Demographic and Health Survey. Rev. salud pública. 2014;16(4):534-546.

11. Ammerman RT, Scheiber FA, Peugh, JL, Messer EP, Van Ginkel JB, Putnam FW. Interpersonal trauma and suicide attempts in lowincome depressed mothers in home visiting. Child Abuse Negl. 2019;97:104126.

12. Al Nasr RS, Altharwi K, Derbah MS, Gharibo SO, Fallatah SA, Alotaibi SG, et al. Prevalence and predictors of postpartum depression in Riyadh, Saudi Arabia: A cross sectional study. PLOS ONE. 2020;15(2):e0228666.

13. Martín-Gómez C, Moreno-Peral P, Bellón JA, Conejo-Cerón S, Campos-Paino H, Gómez-Gómez I, et al. Effectiveness of psychological, psychoeducational and psychosocial interventions to prevent postpartum depression in adolescent and adult mothers: study protocol for a systematic review and meta-analysis of randomised controlled trials. BMJ Open. 2020;10(5):e034424.

14. Cho HJ, Kwon JH, Lee JJ. Antenatal Cognitive-behavioral Therapy for Prevention of Postpartum Depression: A Pilot Study. Yonsei Med J. 2008;49(4):553-562.

15. Tandon SD, Perry DF, Mendelson T, Kemp K, Leis JA. Preventing perinatal depression in low-income home visiting clients: A randomized controlled trial. J Consult Clin Psychol. 2011;79(5):707-712

16. Stein A., Pearson RM, Goodman SH, Rapa E, Rahman A, McCallum M, et al. Effects of perinatal mental disorders on the fetus and child. The Lancet. 2014;384(9956):1800-1819.

17. Guo L, Zhang J, Mu L, Ye Z. Preventing Postpartum Depression With Mindful Self-Compassion Intervention. J Nerv Ment Dis. 2020;208(2):101-107.

18. Maternal depression and child development. J Paediatr Child Health. 2004;9(8):575-598.

19. Beck, CT. Predictors of Postpartum Depression. Nurs Res. 2001;50(5):275-85.

20. Becker M, Weinberger T, Chandy A, \& Schmukler, S. Depression During Pregnancy and Postpartum. Curr Psychiatry Rep. 2016;18(3):32.

21. Robertson E, Grace S, Wallington T, Stewart DE. Antenatal risk factors for postpartum depression: a synthesis of recent literature. Gen Hosp Psychiatry. 2004;26(4):289-95.

22. Committee Opinion No 630. Screening for Perinatal Depression. Obstet Gynecol. 2015;125(5):1268-71.

23. Campo A, Ayola C, Peinado H, Amor M, Cogollo Z. Escala de Edinburgh para depresión posparto: consistencia interna y estructura factorial en mujeres embarazadas de Cartagena, Colombia. Rev Colomb Obstet Ginecol. 2007;58(4):277-83.

24. Chorwe G, Chipps J. A systematic review of screening instruments for depression for use in antenatal services in low resource settings. BMC Psychiatry. 2017;17(1):112.

25. ACOG Committee Opinion No. 757. Obstet Gynecol. 2018;132(5): 208-12.

26. Radloff LS. The CES-D scale a self-report depression scale for research in the general population. Appl Psychol Meas. 1977;1(3):385-401.

27. Mosack V, Shore E. Screening for Depression among Pregnant and 
Postpartum Women. J. Community Health Nurs. 2006;23(1):37-47.

28. Records K, Rice M, \& Beck CT. Psychometric Assessment of the Postpartum Depression Predictors Inventory-Revised. J Nurs Meas. 2007;15(3):189-202.

29. Gorman LL, O'Hara MW, Figueiredo B, Hayes S, Jacquemain F, Kammerer $\mathrm{MH}$, et al. Adaptation of the Structured Clinical Interview for DSM-IV Disorders for assessing depression in women during pregnancy and post-partum across countries and cultures. Br J Psychiatry. 2004;184(S46):s17-s23.

30. Ruyak SL, Qeadan, F. Use of the Antenatal Risk Questionnaire to Assess Psychosocial Risk Factors Associated with Risk for Postpartum Depression: A Pilot Study. J Midwifery Womens Health. 2018; 63:578-83.

31. Werner E, Miller M, Osborne LM, Kuzava S, Monk C. Preventing postpartum depression: review and recommendations. Archv Womens Ment Health. 2014;18(1),41-60.

32. Wang Y, Li H, Peng W, Chen Y, Qiu M, Wang J, et al. Nonpharmacological interventions for postpartum depression. Medicine (Baltimore). 2020;99(31):e21496.

33. Chabrol H, Teissedre F, Saint-Jean M, Teisseyre N, Roge B, Mullet E. Prevention and treatment of post-partum depression: a controlled randomized study on women at risk. Psychol Med. 2002;32(6):1039-47.

34. Cho HJ, Kwon JH, Lee JJ. Antenatal cognitive-behavioral therapy for prevention of postpartum depression: a pilot study. Yonsei Med J. 2008;49(4):553-62.

35. Milgrom J, Schembri C, Ericksen J, Ross J, Gemmill AW. Towards parenthood: an antenatal intervention to reduce depression, anxiety and parenting difficulties. J Affect Disord. 2011;130(3):385-94

36. Stephens S, Ford E, Paudyal P, Smith H. Effectiveness of Psychological Interventions for Postnatal Depression in Primary Care: A Meta-Analysis. Ann Fam Med. 2016;14(5):463-72.

37. Records K, Rice M, Beck CT. Psychometric Assessment of the Postpartum Depression Predictors Inventory-Revised. J Nurs Meas. 2007;15(3):189-202.

38. Gorman LL, O'Hara MW, Figueiredo B, Hayes S, Jacquemain $\mathrm{F}$, Kammerer $\mathrm{MH}$, et al. Adaptation of the Structured Clinical Interview for DSM-IV Disorders for assessing depression in women during pregnancy and post-partum across countries and cultures. Br J Psychiatry Suppl. 2004;184(S46): s17-s23.

39. Duncan LG, Cohn MA, Chao MT, Cook JG, Riccobono J, Bardacke $\mathrm{N}$. Benefits of preparing for childbirth with mindfulness training: a randomized controlled trial with active comparison. BMC Pregnancy Childbirth. 2017;17:140.

40. Bowen A, Baetz M, Schwartz L, Balbuena L, Muhajarine N. Antenatal group therapy improves worry and depression symptoms. Isr J Psychiatry Relat Sci. 2014;51(3):226-31.

41. Sacristan-Martin O, Santed MA, Garcia-Campayo J, Duncan LG, Bardacke N, Fernandez-Alonso C, et al. A mindfulness and compassion-based program applied to pregnant women and their partners to decrease depression symptoms during pregnancy and postpartum: study protocol for a randomized controlled trial. Trials. 2019;20(1):654.

42. Dennis, C.-L., \& Letourneau, N. Global and relationship-specific perceptions of support and the development of postpartum depressive symptomatology. Soc Psychiatry Psychiatr Epidemiol. 2007;42(5), 389-395.

43. Lin N, Ye X, Ensel WM. Social support and depressed mood: a structural analysis. J Health Soc Behav. 1999;40:344-59.

44. Thoits PA. Social support as coping assistance. J Consult Clin Psychol. 1986;54:416-23.

45. ACOG Committee Opinion No. 650: Physical Activity and Exercise During Pregnancy and the Postpartum Period. Obstet Gynecol. 2015;126(6), e135-e142.

46. Ersek JL, Brunner Huber LR. Physical Activity Prior to and During Pregnancy and Risk of Postpartum Depressive Symptoms. J Obstet Gynecol Neonatal Nurs. 2009;38(5):556-66.

47. Da Costa D, Rippen N, Dritsa M, Ring A. Self-reported leisuretime physical activity during pregnancy and relationship to psychological well-being. J Psychosom Obstet Gynaecol. 2003;24(2),111-9.

48. Werner E, Miller M, Osborne LM, Kuzava S, Monk C. Preventing postpartum depression: review and recommendations. Arch Womens Ment Health. 2014;18(1),41-60.

49. Morrell CJ, Sutcliffe P, Booth A, Stevens J, Scope A, Stevenson M, et al. A systematic review, evidence synthesis and meta-analysis of quantitative and qualitative studies evaluating the clinical effectiveness, the cost-effectiveness, safety and acceptability of interventions to prevent postnatal depression. Health Technol Assess. 2016;20(37):1-414.

50. Chabrol H, Teissedre F, Saint-Jean M, Teisseyre N, Rogé B, Mullet E. Prevention and treatment of post-partum depression: a controlled randomized study on women at risk. Psychol Med. 2002;32(6):1039-47

51. Arango C, Díaz-Caneja CM, McGorry PD, Rapoport J, Sommer IE, Vorstman JA, et al. Preventive strategies for mental health. Lancet Psychiatry. 2018;5(7):591-604

52. Bauer A, Knapp M, McDaid D. Assessing the Economic Pay $\square$ off of Low-level Interventions in Reducing Postnatal Depression [Internet]. LSE Health and Social Care; 2012 [citado 2021 mar 22]. Disponible en: http://www.lse.ac.uk/LSEHealthAndSocialCare/ pdf/DP2806.pdf

53. Frieder A, Fersh M, Hainline R, Deligiannidis KM. Pharmacotherapy of postpartum depression: current approaches and novel drug development. CNS Drugs. 2019;33(3):265-82.

54. Yasuma N, Narita Z, Sasaki N, Obikane E, Sekiya J, Inagawa T, et al. Psychological intervention for universal prevention of antenatal and postnatal depression among pregnant women: protocol for a systematic review and meta-analysis. Syst Rev. 2019;8(1):297.

55. Dennis CL, Creedy DK. Psychosocial and psychological interventions for preventing postpartum depression. Cochrane Database Syst Rev. 2004;4:CD001134.

56. Dukhovny D, Dennis CL, Hodnett E, Weston J, Stewart D, Mao W, et al. Prospective economic evaluation of a peer support intervention for prevention of postpartum depression among high-risk women in Ontario, Canada. Am J Perinatol. 2013;30(8):631-42

57. Nakku JE, Nakasi G, Mirembe F. Postpartum major depression at six weeks in primary health care: prevalence and associated factors. Afr Health Sci. 2006;6(4):207-214.

58. Pan WL, Chang CW, Chen SM, Gau ML. Assessing the effectiveness of mindfulness-based programs on mental health during pregnancy and early motherhood - A randomized control trial. BMC Pregnancy Childbirth. 2019;19(1):1-8.

59. Tandon SD, Ward EA, Hamil JL, Jimenez C, Carter M. Perinatal depression prevention through home visitation: a cluster randomized trial of mothers and babies 1-on-1. J Behav Med. 2018;41(5):641-52.

60. Jiang L, Wang ZZ, Qiu LR, Wan GB, Lin Y, Wei Z. Psychological intervention for postpartum depression J. Huazhong Univ. Sci. Technol. [Med. Sci.] 2014;34(3):437-42.

61. Austin MP, Frilingos M, Lumley J, Hadzi-Pavlovic D, Roncolato W, Acland S, et al. Brief antenatal cognitive behaviour therapy group intervention for the prevention of postnatal depression and anxiety: a randomised controlled trial. J Affect Disord. 2008;105(1-3):35-44.

62. Haga SM, Drozd F, Lisøy C, Wentzel-Larsen T, Slinning K. Mamma Mia-A randomized controlled trial of an internetbased intervention for perinatal depression. Psychol Med. 2019;49(11):1850-8.

63. De Vargas C, Rodriguez M, Stein A, Gonçalves B, Garcia D, Pires F, et al. Efficacy of Regular Exercise During Pregnancy on the Prevention of Postpartum Depression: The PAMELA Randomized Clinical Trial. JAMA Netw open. 2019;2(1):e186861.

64. Songøygard KM, Stafne SN, Evensen KAI, Salvesen KA, Vik T, Mørkved S. Does exercise during pregnancy prevent postnatal depression?: A randomized controlled trial. Acta Obstet Gynecol Scand. 2012;91(1):62-7.

65. Dennis CL, Hodnett E, Kenton L, Weston J, Zupancic J, Stewart DE, Kiss A. Effect of peer support on prevention of postnatal depression among high risk women: multisite randomised controlled trial. BMJ. 2009;338:a3064.

66. Fonseca A, Alves S, Monteiro F, Gorayeb R, Canavarro MC. Be a Mom, a web-based intervention to prevent postpartum depression: results from a pilot randomized controlled trial. Behavior Therapy. 2020;51(4):616-33

67. Lara MA, Navarro C, Navarrete L. Outcome results of a psychoeducational intervention in pregnancy to prevent PPD: A randomized control trial. J Affect Disord. 2010;122(1-2):109-17.

68. Ngai FW, Wong PC, Chung KF, Chau PH, Hui PW. Effect of 
couple-based cognitive behavioural intervention on prevention of postnatal depression: multisite randomised controlled trial. BJOG. 2020;127(4):500-7.

69. Vismara L, Sechi C, Lucarelli L. Reflective parenting home visiting program: A longitudinal study on the effects upon depression, anxiety and parenting stress in first-time mothers. Heliyon. 2020;6(7):e04292.

70. Özkan SA, Kücükkelepce DS, Korkmaz B, Yılmaz G, Bozkurt MA. The effectiveness of an exercise intervention in reducing the severity of postpartum depression: A randomized controlled trial. Perspect Psychiatr Care. 2020;56(4):844-50.

71. Sockol LE. A systematic review of the efficacy of cognitive behavioral therapy for treating and preventing perinatal depression. J Affect Disord. 2015;177:7-21.

72. Nagy E, Moore S. Social interventions: An effective approach to reduce adult depression? J Affect Disord. 2017;218:131-152.

73. Horowitz JA, Murphy CA, Gregory K, Wojcik J, Pulcini J, Solon L. Nurse home visits improve maternal/infant interaction and decrease severity of postpartum depression. J Obstet Gynecol Neonatal Nurs. 2013;42(3):287-300.

74. Nakamura A, van der Waerden J, Melchior M, Bolze C, El-Khoury F, Pryor L. Physical activity during pregnancy and postpartum depression: Systematic review and meta-analysis. J Affect Disord. 2019;246:29-41.

75. Pritchett R V., Daley AJ, Jolly K. Does aerobic exercise reduce postpartum depressive symptoms?: A systematic review and meta-analysis. Br J Gen Pract. 2017;67(663):e684-91.

76. Daley AJ, Foster L, Long G, Palmer C, Robinson O, Walmsley H, et al. The effectiveness of exercise for the prevention and treatment of antenatal depression: Systematic review with meta-analysis.
BJOG. 2015;122(1):57-62.

77. Carter T, Bastounis A, Guo B, Morrell CJ. The effectiveness of exercise-based interventions for preventing or treating postpartum depression: a systematic review and meta-analysis. Arch Womens Ment Health. 2019;22(1):37-53.

78. Fontein-Kuipers YJ, Nieuwenhuijze MJ, Ausems M, Budé L, De Vries R. Antenatal interventions to reduce maternal distress: A systematic review and meta-analysis of randomised trials. BJOG. 2014;121(4):389-97.

79. Campolong K, Jenkins S, Clark MM, Borowski K, Nelson N, Moore KM, et al. The association of exercise during pregnancy with trimester-specific and postpartum quality of life and depressive symptoms in a cohort of healthy pregnant women. Arch Womens Ment Health. 2018;21(2):215-24.

80. Shorey S, Ang L, Hon B, Yin C. A systematic mixed-studies review on mindfulness- based childbirth education programs and maternal outcomes. Nursing Outlook. 2019;67(6):696-706.

81. Sheffield KM, Woods-Giscombé CL. Efficacy, feasibility, and acceptability of perinatal yoga on women's mental health and well-being: a systematic literature review. J Holist Nurs. 2016;34(1):64-79.

82. Taylor BL, Cavanagh K, Strauss C. The Effectiveness of MindfulnessBased Interventions in the Perinatal Period: A Systematic Review and Meta-Analysis. PLoS One. 2016;11(5):1-29.

83. Smith CA, Shewamene Z, Galbally M, Schmied V, Dahlen H. The effect of complementary medicines and therapies on maternal anxiety and depression in pregnancy: A systematic review and meta-analysis. J Affect Disord. 2019;245(1):428-39.

84. Gaston A, Cramp A. Exercise during pregnancy: A review of patterns and determinants. J Sci Med Sport. 2011;14(4):299-305. 